\title{
Acoustofluidics 23: Acoustic manipulation combined with other force fields
}

Peter Glynne-Jones, Martyn Hill*

Engineering Sciences, University of Southampton, Southampton, SO17 1BJ, UK.

*E-mail: m.hill@soton.ac.uk; Tel: +44 2380593075

In this, the final paper of the Acoustofluidics series of tutorial articles, we discuss applications in which acoustic radiation forces are used in conjunction with competing or complementary forcefields. This may be in order to enable manipulation operations that would not be easily performed by either force-field alone, or may be used to effect separation based on the different physical principals underlying competing fields. Examples are given of a number of different applications in which acoustic forces are combined with gravitational fields, hydrodynamic forces, electric fields (including dielectrophoresis), magnetic forces and optical forces.

\section{Introduction}

This series of tutorial articles has explored the theory underlying acoustic radiation forces ${ }^{1}$ and acoustically-induced streaming ${ }^{2}$, has described experimental techniques for evaluating these effects ${ }^{3}$, ${ }^{4}$ and has discussed a variety of application $s^{5-8}$ based on both bulk acoustic waves ${ }^{9}$ and surface acoustic waves ${ }^{10}$. It has become apparent that the acoustic phenomena employed have a potentially large scale of action ${ }^{11}$, can trap and manipulate relatively large particles and agglomerates ${ }^{8}$, are appropriate for biological use ${ }^{12}$, and are suitable for integration with many microfluidic fabrication techniques $^{13}$. There are situations in which these properties make the technology attractive for use in combination with other forces, for example when its scale of action complements technologies that generate more localised force fields, or in cases when competition with force fields that scale with other physical properties allows for separation and fractionation.

Integrating acoustophoretic action with other systems within microfluidic devices can create design problems as acoustophoretic systems typically rely on acoustic resonances dependent on the boundaries of the fluid channels, and modifying these boundaries to suit other technologies can interrupt the intended action. In traditional planar resonators ${ }^{9}$ the transducer is usually placed under the channel, with corresponding primary radiation forces out of the plane of the substrate. The in-plane designs of Peterson et $a l^{14,15}$, along with devices actuated by surface acoustic waves ${ }^{10}$, ${ }^{16}$, coupling wedges ${ }^{17}$ or flexural waves ${ }^{18}$ have enabled the acoustic excitation to be placed some distance from the channel, allowing easier integration with other technologies.

For the examples considered here, the way in which the forces scale with particle size and properties is crucial for applications intended to differentiate between particles. For example, in the viscosity dominated regime found in microfluidic applications the Stokes drag force is proportional to particle radius compared to the acoustic radiation force which is proportional to the radius cubed. Thus by 
balancing, or opposing these two forces it is possible to differentiate between particles of different sizes $^{19}$.

With this in mind it is instructive to consider the factors that contribute to the acoustic radiation force $F_{a c}$ on a small sphere ${ }^{1}$ (that is small in comparison to a wavelength) of radius $r$, given by:

$$
F_{a c}(\boldsymbol{r})=\frac{4 \pi r^{3}}{3} \nabla\left(\frac{3\left(\rho_{p}-\rho_{f}\right)}{\left(2 \rho_{p}+\rho_{f}\right)} E_{k i n}(\boldsymbol{r})-\left(1-\frac{\beta_{p}}{\beta_{f}}\right) E_{p o t}(\boldsymbol{r})\right) .
$$

The force is proportional to the volume of the particle and to the gradients of the time averaged kinetic and potential acoustic energy densities $E_{k i n}$ and $E_{p o t}$. The kinetic energy gradient is weighted by a function of the particle and fluid densities $\rho_{p}$ and $\rho_{f}$, while the potential energy gradient is weighted by the particle and fluid compressibilities, $\beta_{p}$ and $\beta_{f}{ }^{20}$. Thus we have the potential to differentiate between particles based on their size, density, and compressibility (in addition, to any other properties to which the second force is sensitive). In practice the effects of varying particle size tend to dominate over the other parameters, which can be problematic if, for example, one wishes to separate two populations of particles whose size distribution overlaps (e.g. separating biological cells).

The following sections describe how different force fields have been used in combination with acoustic forces in a variety of applications. Examples are given of acoustic forces combined with gravitation fields, hydrodynamic forces, electric fields (including dielectrophoresis), magnetic forces and optical forces. At the beginning of each subsection the system properties that influence the complementary force are briefly discussed. For a recent overview of magnetic, dielectrophoretic and optical forces in microfluidic systems, and a comparison with acoustic forces, the reader is referred to Tsutsui and $\mathrm{Ho}^{21}$.

\section{Gravitational Forces}

While levitation against the force of gravity is common in acoustic manipulation, we are concerned here with some example microfluidic systems that utilise the gravitational force in competition with acoustic forces. In equilibrium a particle in an ultrasonic force-field will settle to a position where the gravitational forces (including the buoyancy) balance with the acoustic forces, i.e. when

$$
F_{a c}=\frac{4 \pi r^{3}}{3}\left(\rho_{p}-\rho_{f}\right) g
$$

where $g$ is the gravitational constant. The gravitational force is dependent on the densities of the particle and the fluid and its direction of action relative to other forces can be modified by altering the orientation of the acoustofluidic device. Like the acoustic radiation force, gravitational forces are proportional to particle volume (for homogenous particles), so the equilibrium position is independent of particle size. This size independence can be utilised to provide a method for estimating acoustic energy densities in a manipulation device as described by Spengler et al. ${ }^{22}$. A test particle is placed in the field; as the acoustic field is decreased the particle equilibrium position sinks, until it reaches the turning point (at a position of one eighth of a wavelength below the pressure node, marked ' $O$ ' in Figure 1), where further decrease leads to the particle dropping. Since 
the material properties and hence buoyancy force on the particle are known, the acoustic energy density and hence pressure amplitude can be calculated. However this experiment is prone to high measurement errors due to the difficulty in distinguishing a particle in equilibrium from one that is slowly dropping, and the uncertainty in material properties for the bead. Much more accurate measurements can be produced in lateral aspect devices using PIV based methods ${ }^{23}$. The general presence of the earth's gravitational field makes it a particularly simple field to use yet its relative weakness compared to the acoustic forces in microfluidic systems means that time must be allowed when equilibrium situations have to be reached.

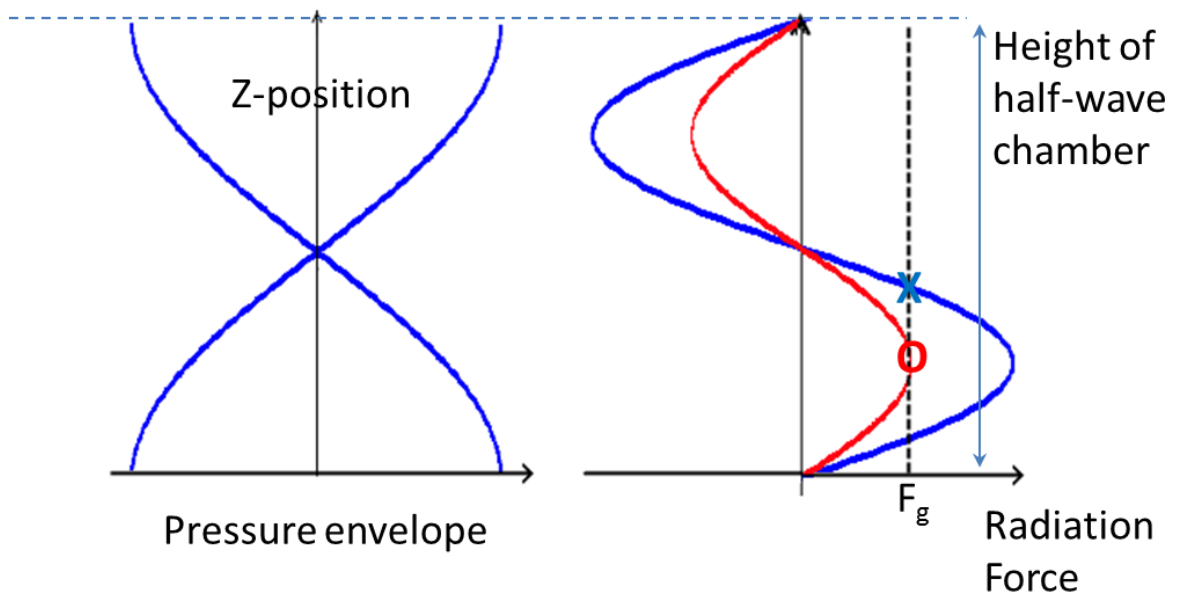

Figure 1. Pressure and force distribution in a 1D standing wave for a half-wave device for two different transducer voltages. For each voltage the cross or circle marks the equilibrium position of a particle with the net gravitational force, $F_{g}$. When performing a 'voltage drop' experiment, the pressure amplitude is steadily reduced until the test particle reaches the height of the position marked with ' $O$ '. At this stage the gravitational forces are balanced by the maximum available force at any height within the fluid, any further reduction in pressure causes the particle to drop. Since this position is independent of particle size, the pressure at the 'drop voltage' can be calculated.

A similar configuration is used by Masudo and Okada ${ }^{24}$, shown here diagrammatically in Figure 2, who tested the acoustic field / displacement relation in detail for a range of different particle types, finding good agreement between experimental results and theoretical predictions. In a later paper ${ }^{25}$ they demonstrate the possibility of sorting particles by their acoustic properties in a size independent manner using the gravity / acoustic field balance. 


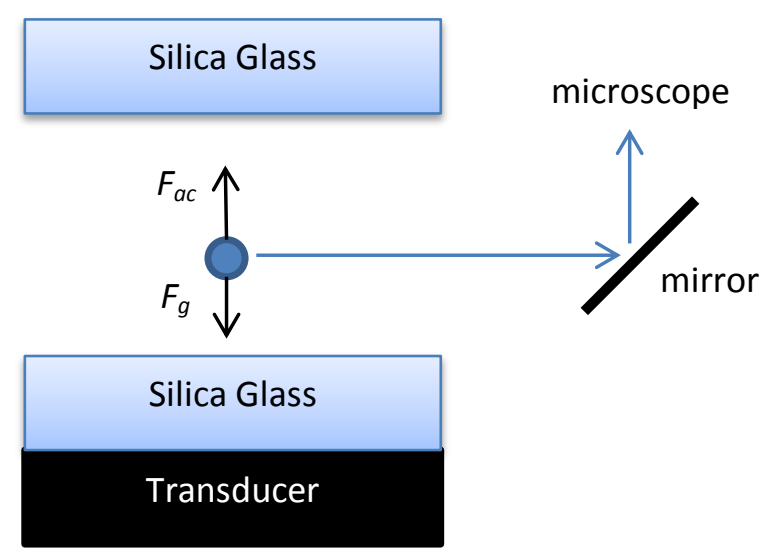

Figure 2. Schematic representation of device used by Masudo and $\mathrm{Okada}^{24}$ to investigate particle separation in a coupled gravity-acoustic field. $F_{a c}$ and $F_{g}$ represent the acoustic and net gravitational forces respectively.

Kanazaki et al. ${ }^{26}$ made use of this relation to monitor ion-exchange processes. An acoustically levitated ion-exchange bead was optically monitored in a system similar to that shown in Figure 2 . In response to a varying chemical environment, counter-ions were replaced in the bead, which led to a change in the physical properties bead including its density and compressibility. Thus the equilibrium position of the bead was directly related to its chemical environment. In contrast to offline sensors, the system offers a compact and dynamically sensitive reading.

The combination of sedimentation and acoustic radiation forces underlie a commercial application of acoustic manipulation technology ${ }^{27,28}$. In continuously perfused bioreactors, acoustic forces are used to aggregate cells such as yeast near the outlet of the reactor. As the aggregates reach a certain critical size, they sediment and drop back into the reactor. In this way the perfusion flow can be maintained without loss of the reactor contents. Similar modes of operation can be found in a range of other work ${ }^{29,30}$.

\section{Hydrodynamic Forces}

Particles within a fluid flow experience a number of hydrodynamic forces. The drag force (dependent on particle size and shape, the fluid flow field, and the fluid viscosity) is discussed here as many particle sorting technologies balance the drag force against the acoustic radiation force. For a sphere of radius $r$ in a low Reynold's number flow, the Stokes drag is

$$
F_{D}=6 \pi \eta r U
$$

where $\eta$ is the dynamic viscosity and $U$ is the relative velocity between the particle and the fluid. In such particle sorting techniques the active section of the devices is too short to allow particles to reach their equilibrium position in the acoustic field (which would be roughly the same for particles of similar density with only a small deviation due to gravitational forces). Typically, particles are initially aligned to a starting position within a flow chamber using a sheath flow ${ }^{14,31,32}$, or an acoustic pre-alignment stage $\mathrm{e}^{33}$, then deflected under the action of the sorting acoustic field which can be excited using bulk axial ${ }^{28}$, bulk lateral ${ }^{29}$, or surface acoustic wave excitation ${ }^{16}$. It can be shown ${ }^{34}$ that in the low Reynolds number regime typically found in this work, particles reach their terminal velocity within a matter of milli-seconds. Since the hydrodynamic drag scales with particle radius (competing with the cubic dependence of radiation force), this terminal velocity (and hence the 
displacement during the time it is in the active region) scales with the square of particle radius. While the acoustic force also depends upon the acoustic properties of the particle, the size dependence will often dominate. Thus when applying this techniques to sorting biological cells, its use is currently restricted to separating cell types whose size distributions do not significantly overlap $^{35}$. A similar principle of competing drag and acoustic forces can be used to separate particles by frequency switching ${ }^{36,37}$, in which the driving frequency of a channel switches between two resonances with a different number of pressure nodes. The time spent at each resonance is chosen so that large particles have time to move to their destination, but the small particles do not move sufficiently far before being pulled back to their original nodal plane as shown in Figure 3

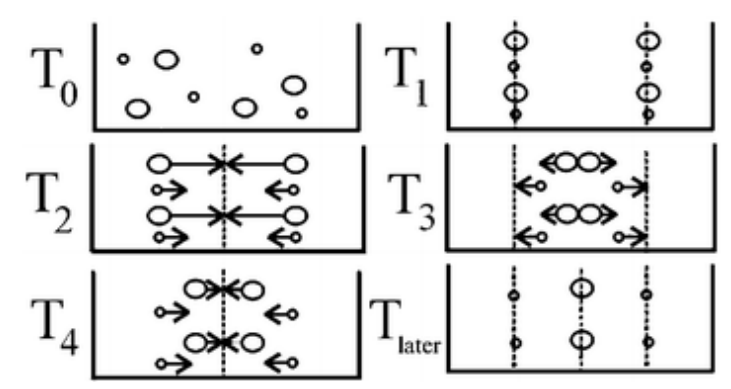

Figure 3. Competing drag and acoustic forces used to separate particles by frequency switching. At $T_{0}$ particles are dispersed and then $\left(T_{1}\right)$ they align at the nodes of the first harmonic. The frequency is switched between the fundamental $\left(T_{2}\right)$ and the first harmonic $\left(T_{3}\right)$ at a rate such that only the largest particles have time to reach the region where at $T_{3}$ exerts a force towards the centre, and hence reach the single node of the fundamental. Reproduced from reference ${ }^{36}$.

Mandralis and Feke ${ }^{19}$ reported the incorporation of acoustic radiation forces into a Field-flow Fractionation (FFF) scheme. In field flow fractionation ${ }^{38}$ a force field is used to cause suspended particles to move into differing positions within a laminar flow field. Particles in regions of higher flow are eluted more quickly than those that remain in slower regions near walls. In this case, two acoustic modes were chosen that drove particles to the centre and wall of the channel respectively. By synchronising an oscillating flow field with the switching between the two acoustic modes, they demonstrated effective separation of a range of polystyrene particles in the range 2-30 $\mu \mathrm{m}$. Bhat and Chakraborty ${ }^{39}$ present a theoretical analysis of acoustic flow-field fractionation subject to the interaction of near-wall attractive and repulsive forces such as electric double-layer fields, and Van Der Waals forces. They predict enhanced sorting resolution under certain combinations of these fields.

Due to boundary and bulk losses, acoustic streaming flow fields are almost universally present in acoustic radiation force devices ${ }^{6}$. The hydrodynamic drag force resulting from this streaming often acts in competition with the radiation force. As above, this effect scales with the square of particle radius, and Bruus has shown ${ }^{34}$ that there is a critical particle radius below which the ultrasonic radiation forces no longer dominate and particle motion is determined primarily by the streaming fields. This phenomenon has been used by Rogers and Neild ${ }^{40}$ to selectively trap particles through a combination of secondary radiation forces (Bjerknes forces) and streaming induced drag forces. In their system, bubbles oscillating in an acoustic field generate strong local streaming currents and attract particles through Bjerknes forces. Depending on their size and density particles are either 
trapped by the Bjerknes forces or carried away from the bubble by the streaming vortex. Figure 4 shows how this was used to selectively trap $5 \mu \mathrm{m}$ silica particles but not $5 \mu \mathrm{m}$ polystyrene particles

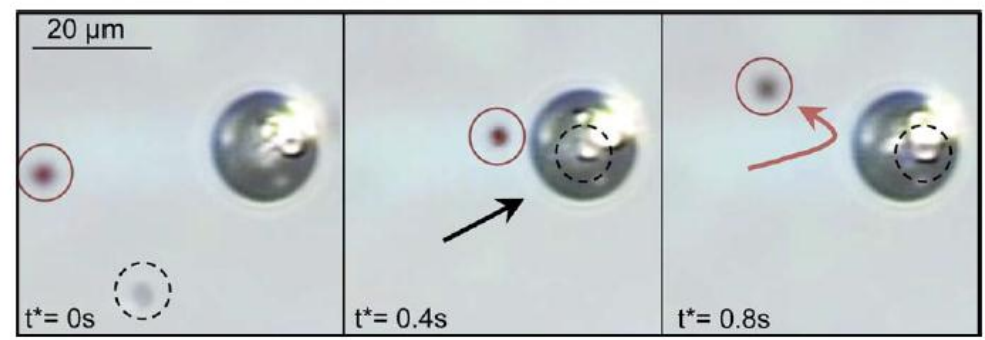

Figure 4. Approach used by Rogers and Neild to selectively trap particles. The grey, silica particles are trapped in contact with the oscillating bubble, while the polystyrene particle (marked in red) circulates within the streaming field. Reproduced from reference ${ }^{40}$.

The balancing of viscous drag forces with acoustic forces can also be used to characterise the forces within a resonant acoustic field. Woodside et al. ${ }^{41}$ estimated the distribution of acoustic energy density within a resonant field by observing the speed of polystyrene particles. They showed how the lateral variations in energy were related to the variation of amplitude across the face of the transducer boundary.

\section{Forces induced by electrical fields}

An approach to particle manipulation based on an electrical field that has been widely exploited in microfluidic systems uses dielectrophoresis (DEP) ${ }^{42}$. The force on a sphere, $F_{D E P}$, depends on the complex permittivities of the particle, $\varepsilon_{p}$, and the fluid medium, $\varepsilon_{f}$, at the angular frequency of operation, $\omega$, such that ${ }^{43}$

$$
F_{D E P}=2 \pi r^{3} \varepsilon_{f} \operatorname{Re}\{K(\omega)\} \nabla|E|^{2},
$$

where $E$ is the r.m.s. electric field and the factor $K(\omega)$ is given by

$$
K(\omega)=\frac{\varepsilon_{p}-\varepsilon_{f}}{\varepsilon_{p}+2 \varepsilon_{f}}
$$

The field induces dipoles in neutral particles by moving the mobile charges associated with them. A gradient in the applied field puts an unequal force on the dipole and moves the particles. The process is effective under $A C$ fields which have the advantage of avoiding electrolysis at the electrodes. DEP can be seen as complementary to ultrasonic manipulation in that DEP performs best in terms of fine control of small numbers of particles over relatively short length scales, while ultrasonic forces can easily act on a large number of particles over rather greater length scales. While DEP is typically sensitive to the conductivity of the fluid medium, with potential heating problems due to electrical currents, acoustic manipulation usually creates less heating due to the low acoustic losses found in most host fluids. Acoustic manipulation is often difficult to control precisely due to the multiple reflection of the waves at surrounding boundaries, while electric fields are much easier to predict from the geometry of their electrodes. 
Wiklund et al. ${ }^{44}$ first demonstrated the integration of both acoustophoretic and DEP particle manipulation within a microfluidic device, as shown in Figure 5. Acoustic standing waves were set up across the width of the channel, to assemble linear arrays and aggregates of particles, which could then be further manipulated by DEP produced by co-planar electrodes at the bottom of the channel. Various manipulation operations were demonstrated. In addition to the possibility of sorting cells through balancing of forces the longer range acoustic forces were used to pre-align populations of cells in preparation for more locally specific DEP switching/manipulation operations.
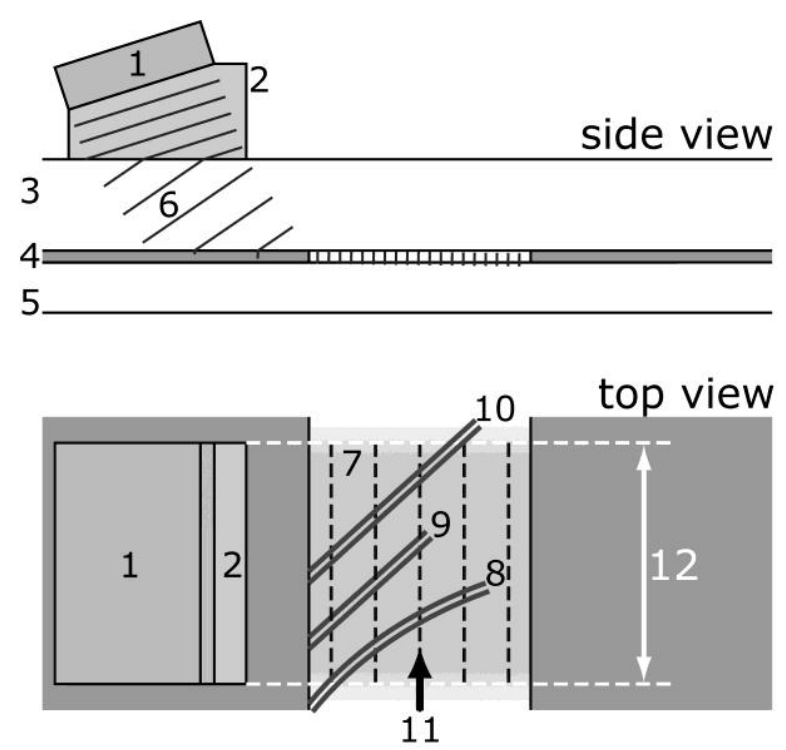

\begin{abstract}
Figure 5. Schematic representation of the combined ultrasonic/DEP system described by Wiklund et al. The piezoceramic transducer (1) is coupled into the structure of the chip (3-5) through a PMMA block (2). The refracted ultrasonic field (6) excites the fluid channel adjacent (12) to the transducer and particles brought into the region by bulk fluid flow (11) are driven to the lateral pressure nodes (7). Hench the particles are pre-aligned before crossing the DEP electrodes (8-10) where combined DEP/ultrasonic manipulation can be implemented. Reproduced from reference ${ }^{44}$.
\end{abstract}

Ravula et al. ${ }^{45}$ describe a layout that utilised the levitation forces generated by ultrasound in a planar resonator coupled to either glass or silicon-substrate DEP chips. Particles are preconcentrated and aligned by the acoustic radiation forces to increase throughput and reduce the positional variability of particles prior to fine adjustments by the DEP electrodes. The authors note that this pre-alignment also reduces the tendency of particles to clump, or to stick to channel walls.

Wiklund et al. ${ }^{46}$ also combined acoustic radiation forces with a capillary electrophoresis (CE) system, to enhance the detection of proteins. The system is designed so that acoustic radiation forces are parallel to the direction of flow, such that viscous drag forces are balanced against the acoustic ones. The electro-osmotic flow (EOF) creates a flow profile that is uniform across the width of the capillary, which ensures that the particle separation is not dependent on lateral particle position. Polystyrene micro-beads functionalised with antibodies indicate the presence of the target protein by forming bead-protein-bead complexes. At the chosen flow rate these complexes tend to be trapped in the acoustic field, while the single beads are carried through by the viscous drag. A CCD detection stage counts the trapped fluorescent beads. 
Neale et al. $^{47}$ describe a combination of DEP and acoustophoresis in which DEP electrodes within a channel immobilise a population of beads into a line which can be further focussed by the acoustic radiation forces into an aggregate. The strength of this combination is in utilising the DEP forces to hold particles against the flow, which although possible acoustically is not easy to achieve or control, while simultaneously using the longer range nature of the acoustic forces to bring the particles to a single focussed location. They also remark on the use of the two alternative forces to effect size dependent sorting. Alternatively, they suggest a 2D sorting approach that fractionates particles into spatial locations that indicate the response of particles to forces from both technologies.

Yasuda et al. ${ }^{48}$ demonstrated a system that allowed electrophoretic forces on particles in water to compete with acoustophoretic ones as shown in Figure 6. Electrodes within a resonant chamber created an electric field of order $10 \mathrm{~V} / \mathrm{mm}$. In the absence of acoustic excitation the effective charge on polystyrene micro-beads was calculated by observing their movement in a low frequency alternating electric field. By then activating the acoustic field, and measuring the position of the beads relative to the pressure node, the acoustic radiation force was determined (in a manner analogous to that described Figure 1, but in this case the electric field replaces the gravitational one), permitting verification of analytical expressions for radiation force.

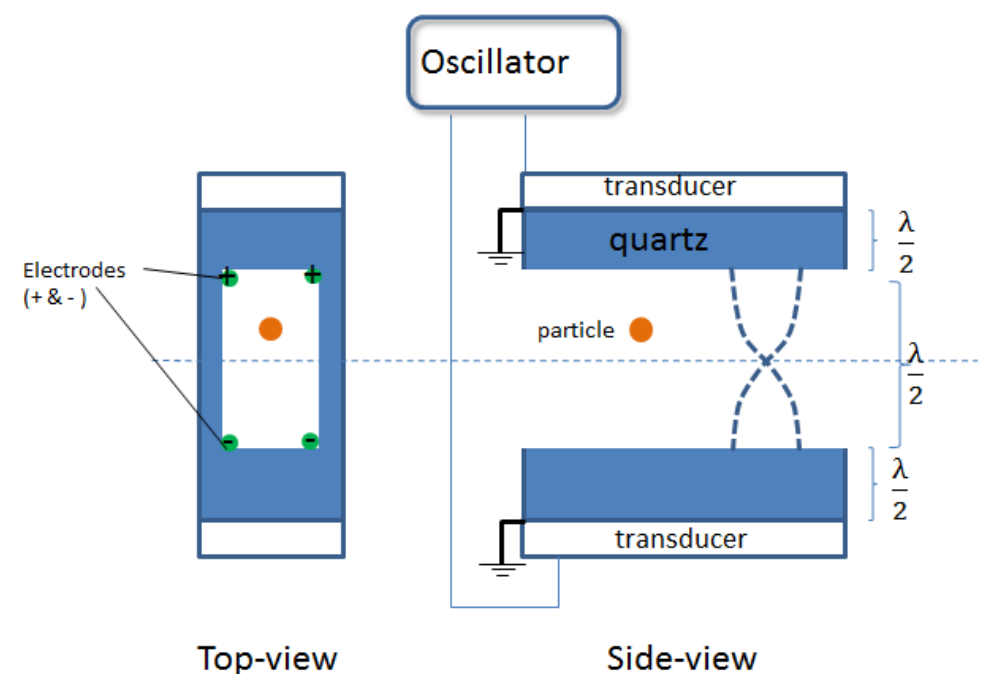

Figure 6. Combined Electrostatic and acoustic forces (after Yasuda et al. ${ }^{48}$ ). Electrostatic forces generated by four electrodes are balanced against acoustic radiation forces arising from a standing wave field, permitting accurate measurement of both types of force.

\section{Magnetic forces}

Magnetic forces, which scale with the difference in susceptibility between the particle, $\chi_{p}$, and the fluid, $\chi_{f}$, have been mentioned previously in this tutorial series in the context of enhanced agglutination ${ }^{49}$ and the force on a sphere in a field of flux density $B$ is ${ }^{50}$

$$
F_{m a g}=2 \pi r^{3} \frac{\left(\chi_{p}-\chi_{f}\right)}{3 \mu_{0}} \nabla|B|^{2},
$$

where $\mu_{0}$ is the magnetic permeability of free space. Adams et al. ${ }^{51}$ demonstrate a microfluidic sorting system that comprises an acoustic sorting stage followed serially by magnetic sorting that 
can operate at rates of up to $10^{8}$ particles per hour. As shown in Figure 7, the input sample is thus separated into three streams: those having low response to both magnetic and acoustic forces, (b) those that respond to acoustic forces only, and (c) those that respond to both magnetic forces and acoustic forces. This could, for example, correspond to separating a sample of cells into streams that correspond to waste material, un-labelled cells, and cells labelled with magnetic beads.

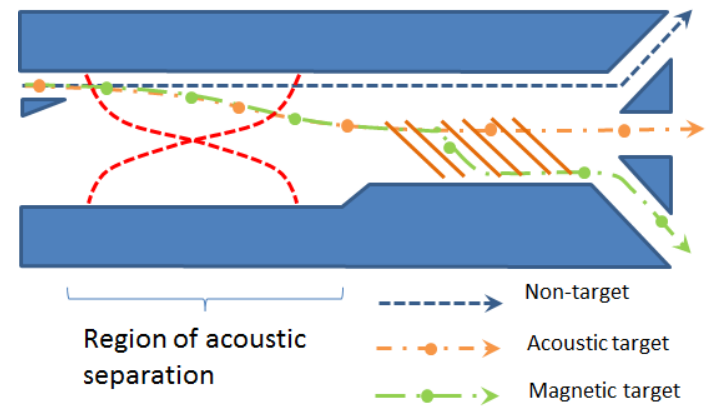

Figure 7: Integrated acoustic and magnetic separation, after Adams et al. ${ }^{51}$. In two successive stages, acoustic then magnetic forces displace particles from their initial position to create three sorted outlets. The red dotted lines indicate the acoustic standing wave field, and orange diagonal elements represent a magnetic comb.

In a device described by Hill et al. ${ }^{52}$, a combination of magnetic, gravitational, and acoustic forces are used to facilitate stages in the sample preparation and concentration of tuberculosis bacteria. Figure 8 shows the device. Initially the bacteria from a sputum sample are labelled with ferrous beads ( $T B$ Beads, supplied by Microsens Biotechnologies), and then a resonant acoustic field is used to create numerous small aggregates of the labelled bacteria/bead complexes; the combination of both acoustic and magnetic forces helps agglomerates to form more rapidly than would occur with magnetism alone. When the acoustic field is switched off the aggregates quickly sediment to the bottom, allowing the supernatant to be washed away. When the acoustic field is reactivated the aggregates are re-suspended, and further washing cycles can take place. Finally, with the acoustic field active, the aggregates can be eluted with a minimum of loss since they are suspended in the centre of the chamber where the flow profile has maximum velocity. The advantage of the acoustic re-suspension over a comparable magnetic stage is that a magnetic process would usually leave the aggregates on a surface, which would cause the aggregate to become disrupted by shearing forces as flow was actuated to remove it.

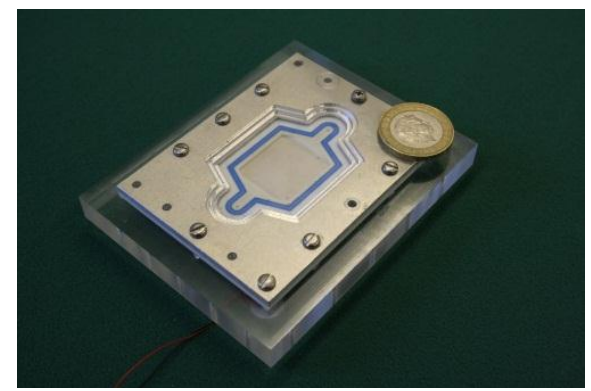

(a)

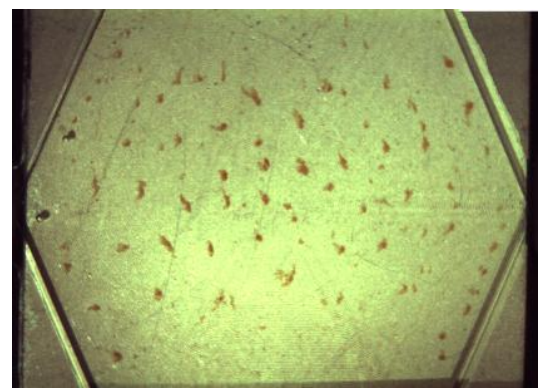

(b)

Figure 8. (a) Triple force unit prototype, utilising magnetic, acoustic and gravitational forces for sample preparation in a TB detection system. Constructed in Macor, glass and aluminium. (b) Acoustically enhanced aggregation of magnetic beads seen in a disposable chamber created entirely from polymeric materials. 


\section{Optical Forces}

Optical traps based on focused laser beams represent another well-established technique for manipulating microparticles and generate a force dependent on the refractive indices of the particle and the fluid medium. When handling particles that are also amenable to acoustic manipulation optical traps will be operating in the Mei regime, where the particle cannot be assumed to be much smaller than a wavelength. However optical gradient forces for small particles in the Rayleigh scattering regime have a similar form to the other gradient forces discussed here. Optical traps, with a wavelength of the order of $1 \mu \mathrm{m}$ are able to manipulate single small particles with a very high degree of precision, while acoustic radiation forces are able to handle a large number of particles simultaneously and can levitate relatively large, dense particles efficiently. Thalhammer et $a l^{53}$ use a combination of acoustical and optical techniques to undertake a variety of manipulation tasks. The ultrasonic field is set up in a square capillary which is held between a glass slide and an optical mirror backed with a PZT transducer, as shown schematically in Figure 9. The mirror allows a laser source, placed below this setup, to act like a dual beam trap ${ }^{54}$, and allows optical manipulation from a large working distance. In this trap, radial optical trapping is due to the lateral gradient force, while axially it is the balancing of the scattering forces. Motile micro-organisms within the fluid in the capillary can be confined within the nodal plane of a half wave acoustic resonance. Once the microorganisms are confined within a plane, an individual organism can be selected, optically trapped, and then moved across the width of the capillary. A further advantage of the combined force fields is that relatively large particles, including a $75 \mu \mathrm{m}$ polystyrene bead and a starch grain, can be manipulated. The force required to levitate the starch grain is of the order of $1 \mathrm{nN}$, which can be achieved comfortably with the acoustic field for a large, high acoustic contrast factor particle. This then allows a relatively low power optical trap to undertake lateral manipulation of the large particles. Finally Thalhammer et al. demonstrate the use of the optical tweezers to measure the trapping forces of the acoustic field at different ultrasonic excitation frequencies.

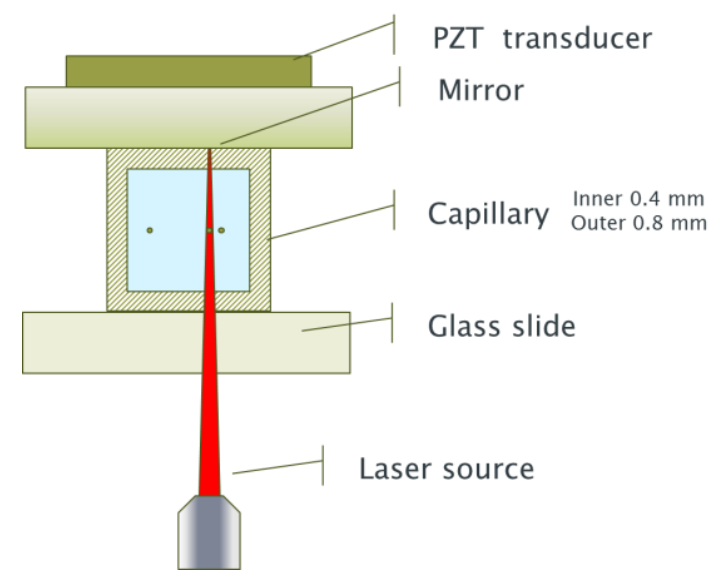

Figure 9. Schematic representation of the setup used by Thalhammer et al. to demonstrate simultaneous acoustic and optical manipulation. A glass capillary is placed between a glass slide and a mirror, on top of which sits a PZT transducer that excites an ultrasonic standing wave in the layered structure. The clear optical path allows a long working distance optical tweezers to further manipulate particles levitated within the ultrasonic field

\section{Conclusions}

As the technologies of acoustofluidics (the combination of microfluidics with ultrasonic particle manipulation) mature there is increasing scope for integrating other force field technologies with it. As with other approaches, acoustofluidic manipulation has operations that it can perform well 
and other operations that benefit from complementary force systems. Acoustofluidics is particularly good for:

- The ability to manipulate large numbers of particles simultaneously over a wide range of sizes and types

- The relatively low complexity of the actuation schemes

- The ability to continuously manipulate cells and maintain viability

- The ability to create multi node traps with simple geometric trapping patterns, including points, lines, planes, and cylinders of trapped particles.

- Creating forces that discriminate on volume, density and compressibility.

Combining these strengths with technologies able to applying forces to single particles among a population, or to discriminate particles on other physical bases will lead to a richer range of microfluidic applications.

\section{References}

1. H. Bruus, Lab on a Chip, 2012, 12, 1014-1021.

2. S. S. Sadhal, Lab on a Chip, 2012, 12, 2292-2300.

3. J. Dual, P. Hahn, I. Leibacher, D. Moller and T. Schwarz, Lab on a Chip, 2012, 12, 852-862.

4. M. Wiklund, H. Brismar and B. Onfelt, Lab on a Chip, 2012, 12, 3221-3234.

5. A. Lenshof, C. Magnusson and T. Laurell, Lab on a Chip, 2012, 12, 1210-1223.

6. M. Wiklund, R. Green and M. Ohlin, Lab on a Chip, 2012, 12, 2438-2451.

7. J. Dual, P. Hahn, I. Leibacher, D. Moller, T. Schwarz and J. Wang, Lab on a Chip, 2012, 12, 4010-4021.

8. M. Evander and J. Nilsson, Lab on a Chip, 2012, 12, 4667-4676.

9. P. Glynne-Jones, R. J. Boltryk and M. Hill, Lab on a Chip, 2012, 12, 1417-1426.

10. M. Gedge and M. Hill, Lab on a Chip, 2012, 12, 2998 - 3007.

11. J. J. Hawkes and S. Radel, Lab on a Chip, 2013, In press.

12. M. Wiklund, Lab on a Chip, 2012, 12, 2018-2028.

13. A. Lenshof, M. Evander, T. Laurell and J. Nilsson, Lab on a Chip, 2012, 12, 684-695.

14. F. Petersson, L. Åberg, A.-M. Swärd-Nilsson and T. Laurell, Anal. Chem., 2007, 79, 5117-5123.

15. F. Petersson, A. Nilsson, C. Holm, H. Jonsson and T. Laurell, Lab On A Chip, 2005, 5, 20-22.

16. J. J. Shi, H. Huang, Z. Stratton, Y. P. Huang and T. J. Huang, Lab on a Chip, 2009, 9, 3354-3359.

17. O. Manneberg, J. Svennebring, H. M. Hertz and M. Wiklund, Journal Of Micromechanics And Microengineering, 2008, 18, 095025.

18. S. Oberti, A. Neild and J. Dual, Journal of the Acoustical Society of America, 2007, 121, 778785.

19. Z. I. Mandralis and D. L. Feke, Aiche Journal, 1993, 39, 197-206.

20. L. P. Gor'kov, Sov. Phys. Dokl., 1962, 6, 773-775.

21. H. Tsutsui and C.-M. Ho, Mechanics Research Communications, 2009, 36, 92-103.

22. J. F. Spengler, M. Jekel, K. T. Christensen, R. J. Adrian, J. J. Hawkes and W. T. Coakley, Bioseparation, 2000, 9, 329-341.

23. P. Augustsson, R. Barnkob, S. T. Wereley, H. Bruus and T. Laurell, Lab on a Chip, 2011, 11, 4152-4164.

24. T. Masudo and T. Okada, Analytical Chemistry, 2001, 73, 3467-3471.

25. T. Masudo and T. Okada, Anal. Sci., 2007, 23, 385-387.

26. T. Kanazaki, S. Hirawa, M. Harada and T. Okada, Analytical Chemistry, 2010, 82, 4472-4478.

27. P. W. S. Pui, F. Trampler, S. A. Sonderhoff, M. Groeschl, D. G. Kilburn and J. M. Piret, Biotechnology Progress, 1995, 11, 146-152. 
28. F. Trampler, J. M. Piret, S. A. Sonderhoff and D. G. Kilburn, United States Pat., 5,626,767, 1997.

29. J. J. Hawkes, M. S. Limaye and W. T. Coakley, J. Appl. Microbiol., 1997, 82, 39-47.

30. M. S. Limaye and W. T. Coakley, J. Appl. Microbiol., 1998, 84, 1035-1042.

31. M. Kumar, D. L. Feke and J. M. Belovich, Biotechnology And Bioengineering, 2005, 89, 129137.

32. J. D. Adams and H. T. Soh, Applied Physics Letters, 2010, 97.

33. P. Augustsson, C. Magnusson, M. Nordin, H. Lilja and T. Laurell, Anal Chem, 2012, 84, 79547962.

34. H. Bruus, Lab on a Chip, 2012, 12, 1578-1586.

35. P. Augustsson, R. Barnkob, C.Grenvall, T. Deierborg, P. Brundin, H. Bruus and T. Laurell, 14th International Conference on Miniaturized Systems for Chemistry and Life Sciences, Groningen, The Netherlands, 2010.

36. T. Laurell, F. Petersson and A. Nilsson, Chemical Society Reviews, 2007, 36, 492-506.

37. Y. Liu and K.-M. Lim, Lab on a Chip, 2011, 11, 3167-3173.

38. J. C. Giddings, K. A. Graff, K. D. Caldwell and M. N. Myers, in Polymer Characterization, American Chemical Society, 1983, vol. 203, ch. 14, pp. 257-269.

39. B. Bhat and S. Chakraborty, Langmuir, 2010, 26, 15035-15043.

40. P. Rogers and A. Neild, Lab on a Chip, 2011, 11, 3710-3715.

41. S. M. Woodside, J. M. Piret, M. Groschl, E. Benes and B. D. Bowen, Aiche Journal, 1998, 44, 1976-1984.

42. A. Castellanos, A. Ramos, A. Gonzalez, N. G. Green and H. Morgan, J. Phys. D-Appl. Phys., 2003, 36, 2584-2597.

43. T. B. Jones, Electromechanics of particles, Cambridge University Press, Cambridge, 1995.

44. M. Wiklund, C. Günther, R. Lemor, M. Jäger, G. Fuhr and H. M. Hertz, Lab on a Chip, 2006, 6, 1537-1544.

45. S. K. Ravula, D. W. Branch, C. D. James, R. J. Townsend, M. Hill, G. Kaduchak, M. Ward and I. Brener, Sensors and Actuators B-Chemical, 2008, 130, 645-652.

46. M. Wiklund, P. Spegel, S. Nilsson and H. M. Hertz, Ultrasonics, 2003, 41, 329-333.

47. S. L. Neale, C. Witte, Y. Bourquin, C. Kremer, A. Menachery, Y. Zhang, R. Wilson, J. Reboud and J. M. Cooper, presented in part at the SPIE 8251, Microfluidics, BioMEMS, and Medical Microsystems X, 2012.

48. K. Yasuda, S. Umemura and K. Takeda, Journal of the Acoustical Society of America, 1996, 99, 1965-1970.

49. M. Wiklund, S. Radel and J. J. Hawkes, Lab on a Chip, 2013, 13, 25-39.

50. M. Zborowski, L. P. Sun, L. R. Moore, P. S. Williams and J. J. Chalmers, J Magn Magn Mater, 1999, 194, 224-230.

51. J. D. Adams, P. Thevoz, H. Bruus and H. T. Soh, Applied Physics Letters, 2009, 95, 254103.

52. M. Hill, P. Glynne-Jones, N. R. Harris, R. J. Boltryk, C. Stanley and D. Bond, presented in part at the Optical Trapping and Optical Micromanipulation, San Diego, 1-5 August 2010, 2010.

53. G. Thalhammer, R. Steiger, M. Meinschad, M. Hill, S. Bernet and M. Ritsch-Marte, Biomed. Opt. Express, 2011, 2, 2859-2870.

54. M. Pitzek, R. Steiger, G. Thalhammer, S. Bernet and M. Ritsch-Marte, Opt. Express, 2009, 17, 19414-19423. 